\title{
A GENERALIZATION OF THE FORELLI-RUDIN CONSTRUCTION AND DEFLATION IDENTITIES
}

\author{
ATSUSHI YAMAMORI \\ (Communicated by Franc Forstneric)
}

\begin{abstract}
We establish a series representation formula of the Bergman kernel of a certain class of domains, which generalizes the Forelli-Rudin construction of the Hartogs domain. Our formula is applied to derive deflation type identities of the Bergman kernels for our domains.
\end{abstract}

\section{INTRODUCTION}

The main purpose of this paper is to generalize the Forelli-Rudin construction [14, which has a direct application to the deflation identity initiated by Boas-FuStraube [1. We start this section with the motivational background.

1.1. Forelli-Rudin construction. Let $D$ be a domain in $\mathbb{C}^{n}$ and $\varphi$ a positive continuous function on $D$. Then the Bergman kernel of $D$ is defined as the reproducing kernel of the space of square integrable holomorphic functions on $D$. As is known from [14] for the Hartogs domain $D_{m, \varphi}=\left\{(z, \zeta) \in D \times \mathbb{C}^{m} ;\|\zeta\|^{2}<\varphi(z)\right\}$, the Bergman kernel $K_{D_{m, \varphi}}$ has the following series representation formula (see also [18]):

$$
K_{D_{m, \varphi}}\left((z, \zeta),\left(z^{\prime}, \zeta^{\prime}\right)\right)=\frac{1}{\pi^{m}} \sum_{k=0}^{\infty}(k+1)_{m} K_{D, \varphi^{k+m}}\left(z, z^{\prime}\right)\left\langle\zeta, \zeta^{\prime}\right\rangle^{k}
$$

Here $K_{D, \varphi^{k+m}}$ is the weighted Bergman kernel of $D$ with respect to $\varphi^{k+m}$ and $(x)_{m}$ is the Pochhammer symbol. Since it was first proved by F. Forelli and W. Rudin [7] for the unit disk $D=\mathbb{D}$ and $\varphi(z)=1-|z|^{2}$, it is called the Forelli-Rudin construction. This formula plays a critical role in establishing explicit formulas of the Bergman kernels of some Hartogs domains (see [21, 222 and [23]). It is also effective in the study of the presence or absence of zeroes of the Bergman kernel, which is called the Lu Qi-Keng problem (see [3] and [24]). In [5], M. Engliš and G. Zhang obtained a generalization of the Forelli-Rudin construction for the following domain:

$$
D_{\varphi}^{\mathcal{F}}=\left\{(z, \zeta) \in D \times \mathbb{C}^{m} ; \varphi(z)^{-1 / 2} \zeta \in \mathcal{F}\right\}
$$

where $\mathcal{F}$ is an irreducible bounded symmetric domain.

Received by the editors April 29, 2013 and, in revised form, July 22, 2013.

2010 Mathematics Subject Classification. Primary 32A25.

The research of the author was supported in part by SRC-GaiA (Center for Geometry and its Applications), the Grant 2011-0030044 from The Ministry of Education, The Republic of Korea. 
In this paper we generalize this result. More precisely, the present paper generalizes the Forelli-Rudin construction to the domains (Theorem 3.1)

$$
D_{P, m}^{\varphi}:=\left\{(z, \zeta) \in D \times \mathbb{C}^{m} ; P\left(\left|\zeta_{1}\right|^{2}, \cdots,\left|\zeta_{m}\right|^{2}\right)<\varphi(z)\right\},
$$

for every quasi-homogeneous function $P$.

1.2. Deflation identity. A generalization of the Forelli-Rudin construction is interesting in its own right. Moreover, we emphasize that our formula enables us to generalize the deflation identity initiated by Boas-Fu-Straube [1, and it provides an additional motivation for our study.

Let $\Omega$ be a bounded domain in $\mathbb{C}^{n}$ which is given by an inequality $\psi(z)<1$. Consider the following two domains:

$$
\begin{aligned}
& \Omega_{1}:=\left\{(z, \zeta) \in \Omega \times \mathbb{C} ;|\zeta|^{2 /\left(q_{1}+q_{2}\right)}<1-\psi(z)\right\}, \\
& \Omega_{2}:=\left\{(z, \zeta) \in \Omega \times \mathbb{C}^{2} ;\left|\zeta_{1}\right|^{2 / q_{1}}+\left|\zeta_{2}\right|^{2 / q_{2}}<1-\psi(z)\right\},
\end{aligned}
$$

where $q_{1}, q_{2}$ are positive real numbers. Then the following identity was proved in [1:

$$
c_{1} K_{\Omega_{1}}\left((z, 0),\left(z^{\prime}, 0\right)\right)=c_{2} K_{\Omega_{2}}\left((z, 0,0),\left(z^{\prime}, 0,0\right)\right),
$$

where $c_{1}$ and $c_{2}$ are constants. This identity is called the deflation identity. The proof is based on the fact that both sides of (1.2) represent the same weighted

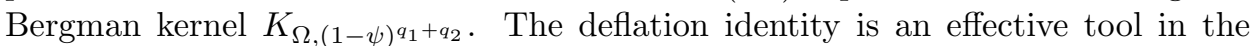
study of the Lu Qi-Keng problem (for details, see [1]).

In the case of the Hartogs domains, due to the Forelli-Rudin construction, the restriction of $K_{D_{m, \varphi}}$ to the subspace $\left\{\zeta, \zeta^{\prime}=0\right\}$ coincides with $K_{D, \varphi^{m}}\left(z, z^{\prime}\right)$ (up to a constant). This kind of property also holds for our domain $D_{P, m}^{\varphi}$ and it allows us to study deflation identities for $D_{P, m}^{\varphi}$.

The deflation identity (1.2) relates the Bergman kernels of domains in different dimensions, while our deflation identity also relates those of domains in the same

dimension. For instance, the restrictions of the Bergman kernels $K_{\Omega_{1}}, K_{\Omega_{2}}$ to the subspace $\left\{\zeta, \zeta^{\prime}=0\right\}$ coincides, up to a constant multiple, with those of the following domains $\Omega_{3}, \Omega_{4}$ :

$$
\begin{aligned}
& \Omega_{3}:=\left\{(z, \zeta) \in \Omega \times \mathbb{C}^{2} ;\left|\zeta_{1}\right|^{2}+\left|\zeta_{2}\right|^{2 / q_{2}}<(1-\psi(z))^{\frac{q_{1}+q_{2}}{1+q_{2}}}\right\}, \\
& \Omega_{4}:=\left\{(z, \zeta) \in \Omega \times \mathbb{C}^{2} ;\left|\zeta_{1}\right|^{2 / q_{1}}+\left|\zeta_{2}\right|^{2 / q_{1}}<(1-\psi(z))^{\frac{q_{1}+q_{2}}{2 q_{1}}}\right\} .
\end{aligned}
$$

1.3. Organization of the paper. Subsequent to this introduction, Section 2 provides some basic properties of the Bergman kernel and the Reinhardt domain $D_{P}^{m}(r)$. Using the results given in Section 2, we prove our main result (Theorem 3.1) in Section 3. In Section 4 by using our Forelli-Rudin type formula, we study deflation identities for $D_{P, m}^{\varphi}$.

\section{Preliminaries}

2.1. Bergman kernel. In this section, we collect some basic properties of the Bergman kernel. Let $D$ be a domain in $\mathbb{C}^{n}, \varphi$ a positive continuous function on $D$ and $L_{a}^{2}(D, \varphi)$ the Hilbert space of square integrable holomorphic functions with respect to the weight function $\varphi$ on $D$ with the inner product

$$
\langle f, g\rangle=\int_{D} f(z) \overline{g(z)} \varphi(z) d V(z), \quad \text { for all } f, g \in \mathcal{O}(D) .
$$


The weighted Bergman kernel $K_{D, \varphi}$ of $D$ with respect to the weight $\varphi$ is the reproducing kernel of $L_{a}^{2}(D, \varphi)$. Namely, the weighted Bergman kernel $K_{D, \varphi}$ has the following reproducing property:

$$
f(z)=\int_{D} K_{D, \varphi}(z, w) f(w) \varphi(w) d V(w), \quad \text { for all } f \in L_{a}^{2}(D, \varphi) .
$$

If $\varphi \equiv 1$, the reproducing kernel is called the (unweighted) Bergman kernel. Let $f: D_{1} \rightarrow D_{2}$ be a biholomorphic map between two domains $D_{1}$ and $D_{2}$ in $\mathbb{C}^{n}$. Then the following transformation law is known:

$$
K_{D_{1}}(z, w)=\operatorname{det} f^{\prime}(z) K_{D_{2}}(f(z), f(w)) \overline{\operatorname{det} f^{\prime}(w)},
$$

for all $z, w \in D_{1}$, where $f^{\prime}(z)$ is the complex Jacobian of $f$. Let $\left\{f_{\alpha}\right\}$ be a complete orthonormal basis for $L_{a}^{2}(D, \varphi)$; then the kernel $K_{D, \varphi}$ is computed by

$$
K_{D, \varphi}(z, w)=\sum_{\alpha} f_{\alpha}(z) \overline{f_{\alpha}(w)},
$$

which is independent of the choice of basis. Further information about the Bergman kernel can be found in [10, Chapter 3] and [13, Chapter 1].

2.2. Reinhardt domain $D_{P}^{m}(r)$. In this section we study a certain Reinhardt domain $D_{P}^{m}(r)$. We start this section with basic definitions.

Definition 2.1. A domain $D$ in $\mathbb{C}^{m}$ is called a Reinhardt domain if it is invariant under the rotations:

$$
\left(\zeta_{1}, \cdots, \zeta_{m}\right) \mapsto\left(e^{i \theta_{1}} \zeta_{1}, \cdots, e^{i \theta_{m}} \zeta_{m}\right), \quad\left(\theta_{1}, \cdots, \theta_{m}\right) \in \mathbb{R}^{m}
$$

Moreover a Reinhardt domain $D$ is called complete if, whenever the point $\left(\zeta_{1}, \cdots\right.$, $\left.\zeta_{m}\right) \in D$, then $\left(c_{1} \zeta_{1}, \cdots, c_{m} \zeta_{m}\right) \in D$ for all complex constants $c_{j}$ satisfying $\left|c_{j}\right| \leq 1$ for all $j=1, \cdots, m$.

Definition 2.2. Let $P$ be a real continuous function on $\mathbb{R}^{m}$. Assume that, for all $\lambda \geq 0$, there exist positive real numbers $\alpha_{1}, \cdots, \alpha_{m}$ such that

$$
\lambda P\left(x_{1}, \cdots, x_{m}\right)=P\left(\lambda^{\alpha_{1}} x_{1}, \cdots, \lambda^{\alpha_{m}} x_{m}\right),
$$

for all $\left(x_{1}, \cdots, x_{m}\right) \in \mathbb{R}^{m}$. A function $P$ satisfying this condition is called quasihomogeneous with weight $\alpha$. In particular, a quasi-homogeneous function is called homogeneous if $\alpha_{i}=1$ for all $1 \leq i \leq m$.

In this section, we consider the following Reinhardt domain $D_{P}^{m}(r)$ :

$$
D_{P}^{m}(r):=\left\{\zeta \in \mathbb{C}^{m} ; P\left(\left|\zeta_{1}\right|^{2}, \cdots,\left|\zeta_{m}\right|^{2}\right)<r^{2}\right\}, \quad r>0
$$

with the conditions

(i) $P$ is quasi-homogeneous with weight $\alpha$,

(ii) $D_{P}^{m}(r)$ is bounded and complete.

The aim of this section is to give a relation between $\left\|\zeta_{1}^{k_{1}} \cdots \zeta_{m}^{k_{m}}\right\|_{L_{a}^{2}\left(D_{P}^{m}(r)\right)}^{2}$ and $\left\|\zeta_{1}^{k_{1}} \cdots \zeta_{m}^{k_{m}}\right\|_{L_{a}^{2}\left(D_{P}^{m}(1)\right)}^{2}$ (Proposition 2.1).

By condition (ii), we know that $(0, \cdots, 0) \in D_{P}^{m}(r)$. This, together with (ii) implies that the domain $D_{P}^{m}(r)$ is a complete Reinhardt domain with center at the origin. It is well known that for any complete Reinhardt domain $D$ with center at the origin, the set of (normalized) monomials forms a complete orthonormal basis 
of $L_{a}^{2}(D)$ (see [17, Exercise 8.8]). Thus the Bergman kernel $K_{D_{P}^{m}(r)}$ of $D_{P}^{m}(r)$ is given by

$$
K_{D_{P}^{m}(r)}\left(\zeta, \zeta^{\prime}\right)=\sum_{k \in \mathbb{N}^{m}} a_{k_{1} \cdots k_{m}}(r)\left(\zeta \overline{\zeta \zeta^{\prime}}\right)^{k}
$$

where $\left(\zeta \overline{\zeta^{\prime}}\right)^{k}=\left(\zeta_{1} \overline{\zeta_{1}^{\prime}}\right)^{k_{1}} \cdots\left(\zeta_{m} \overline{\zeta_{m}^{\prime}}\right)^{k_{m}}$ and the coefficient $a_{k_{1} \cdots k_{m}}(r)$ is given by

$$
\begin{aligned}
a_{k_{1} \cdots k_{m}}(r) & =\left\|\zeta_{1}^{k_{1}} \cdots \zeta_{m}^{k_{m}}\right\|_{L_{a}^{2}\left(D_{P}^{m}(r)\right)}^{-2} \\
& =\left(\int_{D_{P}^{m}(r)}\left|\zeta_{1}^{k_{1}} \cdots \zeta_{m}^{k_{m}}\right|^{2} d \zeta\right)^{-1} .
\end{aligned}
$$

In the case of $r=1$, for simplicity of notation, we put $a_{k_{1} \cdots k_{m}}=a_{k_{1} \cdots k_{m}}$ (1). By condition (ii), we know that

$$
\begin{aligned}
D_{P}^{m}(r) & =\left\{\zeta \in \mathbb{C}^{m} ; P\left(\left|\zeta_{1}\right|^{2}, \cdots,\left|\zeta_{m}\right|^{2}\right)<r^{2}\right\} \\
& =\left\{\zeta \in \mathbb{C}^{m} ; P\left(\frac{\left|\zeta_{1}\right|^{2}}{r^{2 \alpha_{1}}}, \cdots, \frac{\left|\zeta_{m}\right|^{2}}{r^{2 \alpha_{m}}}\right)<1\right\} .
\end{aligned}
$$

It implies that there is a biholomorphic mapping $F$ from $D_{P}^{m}(r)$ to $D_{P}^{m}(1)$ :

$$
F: D_{P}^{m}(r) \rightarrow D_{P}^{m}(1),\left(\zeta_{1}, \cdots, \zeta_{m}\right) \mapsto\left(\frac{\zeta_{1}}{r^{\alpha_{1}}}, \cdots, \frac{\zeta_{m}}{r^{\alpha_{m}}}\right) .
$$

By the transformation law of the Bergman kernel (2.1) and (2.4), it follows that

$$
K_{D_{P}^{m}(r)}\left(\zeta, \zeta^{\prime}\right)=\frac{1}{r^{2|\alpha|}} \sum_{k \in \mathbb{N}^{m}} a_{k_{1} \cdots k_{m}}\left(\frac{\zeta_{1} \overline{\zeta^{\prime} 1}}{r^{2 \alpha_{1}}}\right)^{k_{1}} \cdots\left(\frac{\zeta_{m} \overline{\zeta_{m}^{\prime} m}}{r^{2 \alpha_{m}}}\right)^{k_{m}} .
$$

Comparing the above two expansions (2.4) and (2.5) of $K_{D_{P}^{m}(r)}$, we obtain a relation:

$$
\left\|\zeta_{1}^{k_{1}} \cdots \zeta_{m}^{k_{m}}\right\|_{L_{a}^{2}\left(D_{P}^{m}(r)\right)}^{2}=r^{2|\alpha(1+k)|}\left\|\zeta_{1}^{k_{1}} \cdots \zeta_{m}^{k_{m}}\right\|_{L_{a}^{2}\left(D_{P}^{m}(1)\right)}^{2},
$$

where we put $|\alpha(1+k)|=\sum_{i=1}^{m} \alpha_{i}\left(1+k_{i}\right)$. In summary, we showed the following proposition:

Proposition 2.1. For any positive real number $r$, we have the following relation:

where $|\alpha(1+k)|=\sum_{i=1}^{m} \alpha_{i}\left(1+k_{i}\right)$.

$$
\left\|\zeta_{1}^{k_{1}} \cdots \zeta_{m}^{k_{m}}\right\|_{L_{a}^{2}\left(D_{P}^{m}(r)\right)}^{2}=r^{2|\alpha(1+k)|}\left\|\zeta_{1}^{k_{1}} \cdots \zeta_{m}^{k_{m}}\right\|_{L_{a}^{2}\left(D_{P}^{m}(1)\right)}^{2},
$$

Now we give some examples.

Example 2.3. Let us consider the case $P\left(x_{1}, \cdots, x_{m}\right)=x_{1}+\cdots+x_{m}$. In this case, $\alpha_{1}=\cdots=\alpha_{m}=1$ and the domain $D_{P}^{m}(r)$ coincides with the unit ball of radius $r$ :

$$
D_{P}^{m}(r)=\left\{\zeta \in \mathbb{C}^{m} ;\|\zeta\|^{2}<r^{2}\right\}=: \mathbb{B}^{m}(r)
$$

It follows that

$$
\left\|\zeta_{1}^{k_{1}} \cdots \zeta_{m}^{k_{m}}\right\|_{L_{a}^{2}\left(\mathbb{B}^{m}(r)\right)}^{2}=r^{2(m+|k|)}\left\|\zeta_{1}^{k_{1}} \cdots \zeta_{m}^{k_{m}}\right\|_{L_{a}^{2}\left(\mathbb{B}^{m}(1)\right)}^{2} .
$$

The coefficient $a_{k_{1} \cdots k_{m}}$ has the following explicit form:

$$
a_{k_{1} \cdots k_{m}}=\frac{(|k|+m) !}{\pi^{m} k_{1} ! \cdots k_{m} !} .
$$


Example 2.4. Let $q_{1}, \cdots, q_{m}$ be positive integers. We now consider the case $P\left(x_{1}, \cdots, x_{m}\right)=x_{1}^{q_{1}}+\cdots+x_{m}^{q_{m}}$. In this case, we know that $\alpha_{i}=q_{i}^{-1}$ and

$$
D_{P}^{m}(r)=\left\{\zeta \in \mathbb{C}^{m} ;\left|\zeta_{1}\right|^{2 q_{1}}+\cdots+\left|\zeta_{m}\right|^{2 q_{m}}<r^{2}\right\}=: D_{q}(r) .
$$

The domain $D_{q}(r)$ is called a complex ellipsoid. Then we have

$$
\left\|\zeta_{1}^{k_{1}} \cdots \zeta_{m}^{k_{m}}\right\|_{L_{a}^{2}\left(D_{q}(r)\right)}^{2}=r^{2\left|q^{-1}(1+k)\right|}\left\|\zeta_{1}^{k_{1}} \cdots \zeta_{m}^{k_{m}}\right\|_{L_{a}^{2}\left(D_{q}(1)\right)}^{2} .
$$

It is known that $a_{k_{1} \cdots k_{m}}$ has the following expression (see [2]):

$$
a_{k_{1} \cdots k_{m}}=\frac{q_{1} \cdots q_{m} \Gamma\left(1+\sum_{j=1}^{m}\left(k_{j}+1\right) / q_{j}\right)}{\pi^{m} \prod_{j=1}^{m} \Gamma\left(\left(k_{j}+1\right) / q_{j}\right)} .
$$

Example 2.5. Let $q_{1}, \cdots, q_{m}$ be positive real numbers. We consider the case $P\left(x_{1}, \cdots, x_{m}\right)=\sum_{i=1}^{m} x_{i}^{1 / q_{i}}+\prod_{i=1}^{m} x_{i}^{\frac{1}{m q_{i}}}$. Then we have

$$
D_{P}^{m}(r)=\left\{\zeta \in \mathbb{C}^{m} ; \sum_{i=1}^{m}\left|\zeta_{i}\right|^{2 / q_{i}}+\prod_{i=1}^{m}\left|\zeta_{i}\right|^{\frac{2}{m q_{i}}}<r^{2}\right\} .
$$

In this case, we know that $\alpha_{i}=q_{i}$ and

$$
\left\|\zeta_{1}^{k_{1}} \cdots \zeta_{m}^{k_{m}}\right\|_{L_{a}^{2}\left(D_{P}^{m}(r)\right)}^{2}=r^{2|q(1+k)|}\left\|\zeta_{1}^{k_{1}} \cdots \zeta_{m}^{k_{m}}\right\|_{L_{a}^{2}\left(D_{P}^{m}(1)\right)}^{2} .
$$

Remark 2.6. It is known that the Bergman kernel of $D_{q}(r)$ is expressed in terms of hypergeometric functions (see [8]). In particular, J.-D. Park proved that the Bergman kernel of the 2-dimensional complex ellipsoid

$$
D_{\left(q_{1}, q_{2}\right)}=\left\{\left(z_{1}, z_{2}\right) \in \mathbb{C}^{2} ;\left|z_{1}\right|^{2 q_{1}}+\left|z_{2}\right|^{2 q_{2}}<1\right\}
$$

is represented by means of elementary functions if and only if $q=\left(1, q_{2}\right),\left(q_{1}, 1\right),(2,2)$ and obtained the Bergman kernel in explicit form when $q=(2,2)$ (see [15]). Recently, J.-D. Park also obtained a closed form of the Bergman kernel of the 3dimensional complex ellipsoid [16]:

$$
D_{(4,4,4)}=\left\{\left(z_{1}, z_{2}, z_{3}\right) \in \mathbb{C}^{3} ;\left|z_{1}\right|^{4}+\left|z_{2}\right|^{4}+\left|z_{3}\right|^{4}<1\right\} .
$$

We note that condition (ii) is closely related to bounded Reinhardt domains with non-compact automorphism group and $C^{k}$-smooth boundary, $k \geq 1$. Indeed, the following result is known (cf. [11] and [12):

Theorem 2.7. Let $D \subset \mathbb{C}^{n}$ be a bounded Reinhardt domain with $C^{k}$-smooth boundary, and if $A u t(D)$ is not compact, then, up to dilations and permutations of coordinates, $D$ is a domain of the form

$$
\left\{\left(z_{1}, \cdots, z_{n}\right) \in \mathbb{C}^{n} ;\left|z_{1}\right|^{2}+\psi\left(\left|z_{2}\right|, \ldots,\left|z_{n}\right|\right)<1\right\},
$$

where $\psi$ is a non-negative $C^{k}$-smooth function in $\mathbb{R}^{n-1}$ that is strictly positive in $\mathbb{R}^{n-1} \backslash\{0\}$ and such that $\psi\left(\left|z_{2}\right|, \cdots,\left|z_{n}\right|\right)$ is $C^{k}$-smooth in $\mathbb{C}^{n-1}$, and

$$
\psi\left(t^{\frac{1}{\alpha_{1}}} x_{2}, \cdots, t^{\frac{1}{\alpha_{n}}} x_{n}\right)=t \psi\left(x_{2}, \cdots, x_{n}\right),
$$

in $\mathbb{R}^{n-1}$ for all $t \geq 0$. Here $\alpha_{j}>0, j=2, \cdots, n$, and each $\alpha_{j}$ is either an even integer or $\alpha_{j}>2 k$.

We also remark that a classification of Reinhardt domains with a description of their automorphism groups was obtained by Shimizu [19] and Sunada [20] (see also [10. Chapter 8]). We close this section with one more remark on condition (ii). 
Remark 2.8. Consider a function $P$ on $\mathbb{R}^{2}$ with the following condition:

$$
h(\lambda) P\left(x_{1}, x_{2}\right)=P\left(\lambda^{\alpha_{1}} x_{1}, \lambda^{\alpha_{2}} x_{2}\right),
$$

for all $x \in \mathbb{R}^{2}$ and $\lambda>0$. Here $h$ is a given continuous function. Then $h$ can be written in the form $h(\lambda)=\lambda^{r}$ for some $r \in \mathbb{R}$. Indeed, it can be shown by checking $h\left(\lambda_{1} \lambda_{2}\right)=h\left(\lambda_{1}\right) h\left(\lambda_{2}\right)$ for all $\lambda_{1}, \lambda_{2}>0$.

\section{Generalized Forelli-Rudin construction}

Let $D$ be a domain $\in \mathbb{C}^{n}$ and $\varphi$ be a positive continuous function on $D$. In this section, we consider the following domain:

$$
D_{P, m}^{\varphi}:=\left\{(z, \zeta) \in D \times \mathbb{C}^{m} ; P\left(\left|\zeta_{1}\right|^{2}, \cdots,\left|\zeta_{m}\right|^{2}\right)<\varphi(z)\right\}
$$

with the same assumptions (ii), (iii). In the previous section, using condition (ii), we showed Proposition 2.1 for $D_{P}^{m}(r)$. Proposition 2.1 gives us a relation between the Bergman kernel of $D_{P, m}^{\varphi}$ and the weighted Bergman kernels of the base domain $D$.

3.1. Main result. As is shown in [18, Proposition 1.1], for the Hartogs domain

$$
\widehat{D}=\left\{(z, \zeta) \in D \times \mathbb{C} ;|\zeta|^{2}<\varphi(z)\right\},
$$

the set $S=\left\{(k+1)^{1 / 2} f_{j, k+1}(z) \zeta^{k}\right\}_{k \in \mathbb{N}, j \in J_{k+1}}$ is a complete orthonormal basis of $L_{a}^{2}(\widehat{D})$. Here $\left\{f_{j, k}\right\}_{j \in J_{k}}$ is a complete orthonormal basis of $L_{a}^{2}\left(D, \varphi^{k}\right)$. This fact, together with (2.2), implies the Forelli-Rudin construction for $\widehat{D}$. Our proof of the main theorem is based on the idea mentioned above.

Now we state the main theorem.

Theorem 3.1. The Bergman kernel $K_{D_{P, m}^{\varphi}}$ of $D_{P, m}^{\varphi}$ has the following series representation:

$$
K_{D_{P, m}^{\varphi}}\left((z, \zeta),\left(z^{\prime}, \zeta^{\prime}\right)\right)=\sum_{k \in \mathbb{N}^{m}} a_{k_{1} \cdots k_{m}} K_{D, \varphi|\alpha(1+k)|}\left(z, z^{\prime}\right)\left(\zeta \overline{\zeta \zeta^{\prime}}\right)^{k}
$$

Here $a_{k_{1} \cdots k_{m}}=\left\|\zeta_{1}^{k_{1}} \cdots \zeta_{m}^{k_{m}}\right\|_{L_{a}^{2}\left(D_{P}^{m}(1)\right)}^{-2}$.

Proof. Let $f \in L_{a}^{2}\left(D_{P, m}^{\varphi}\right)$. By the Taylor expansion, with respect to the variable $\zeta$, we have

$$
f(z, \zeta)=\sum_{k \in \mathbb{N}^{m}} b_{k}(z) \zeta_{1}^{k_{1}} \cdots \zeta_{m}^{k_{m}}
$$

Here the function $b_{k}(z)$ is holomorphic in $z$ and the series converges uniformly on compact subsets of $D_{p, m}^{\varphi}$. Let us compute $\|f\|_{L_{a}^{2}\left(D_{P, m}^{\varphi}\right)}^{2}$ :

$$
\begin{aligned}
& \infty>\|f\|_{L_{a}^{2}\left(D_{P, m}^{\varphi}\right)}^{2}=\lim _{\epsilon \rightarrow 0} \int_{D_{P, m}^{\varphi-\epsilon}}|f(z, \zeta)|^{2} d z d \zeta \\
& =\lim _{\epsilon \rightarrow 0} \sum_{k \in \mathbb{N}^{m}} \int_{z \in D}\left|b_{k}(z)\right|^{2}\left(\int_{D_{P}^{m}(\sqrt{(\varphi-\epsilon)})}\left|\zeta_{1}^{k_{1}} \cdots \zeta_{m}^{k_{m}}\right|^{2} d \zeta\right) d z \\
& =\lim _{\epsilon \rightarrow 0} \sum_{k \in \mathbb{N}^{m}} a_{k_{1} \cdots k_{m}}^{-1} \int_{z \in D}\left|b_{k}(z)\right|^{2}(\varphi(z)-\epsilon)^{|\alpha(1+k)|} d z \\
& =\sum_{k \in \mathbb{N}^{m}} a_{k_{1} \cdots k_{m}}^{-1}\left\|b_{k}(z)\right\|_{L_{a}^{2}(D, \varphi|\alpha(1+k)|)}^{2} .
\end{aligned}
$$


Here the third equality follows from Proposition 2.1. Thus if $f \in L_{a}^{2}\left(D_{P, m}^{\varphi}\right)$, then $b_{k}(z) \in L_{a}^{2}\left(D, \varphi^{|\alpha(1+k)|}\right)$. For all $h \in L_{a}^{2}\left(D, \varphi^{|\alpha(1+k)|}\right)$, we have

$$
\begin{aligned}
\left\|h(z) \zeta_{1}^{k_{1}} \cdots \zeta_{m}^{k_{m}}\right\|_{L_{a}^{2}\left(D_{P, m}^{\varphi}\right)}^{2} & =\int_{z \in D}|h(z)|^{2}\left(\int_{D_{P}^{m}(\sqrt{\varphi(z)})}\left|\zeta_{1}^{k_{1}} \cdots \zeta_{m}^{k_{m}}\right|^{2} d \zeta\right) d z \\
& \left.=a_{k_{1} \cdots k_{m}}^{-1}\|h(z)\|_{L_{a}^{2}(D, \varphi|\alpha(1+k)|}^{2}\right) \\
& <\infty
\end{aligned}
$$

Let $\left\{e_{j k}\right\}_{j \in J_{k}}$ be a complete orthonormal basis of $L_{a}^{2}\left(D, \varphi^{|\alpha(1+k)|}\right)$. Put $\zeta^{k}=$ $\zeta_{1}^{k_{1}} \cdots \zeta_{m}^{k_{m}}$. Then $e_{j k} \zeta^{k}$ is an element of $L_{a}^{2}\left(D_{P, m}^{\varphi}\right)$ for all $k \in \mathbb{N}^{m}, j \in J_{k}$. Now we show that $S=\left\{a_{k_{1} \cdots k_{m}}^{1 / 2} e_{j k} \zeta^{k}\right\}_{j \in J_{k}, k \in \mathbb{N}^{m}}$ is a complete orthonormal basis of $L_{a}^{2}\left(D_{P, m}^{\varphi}\right)$. Put $g_{j k}=a_{k_{1} \cdots k_{m}}^{1 / 2} e_{j k} \zeta^{k}$ (resp. $\left.g_{j^{\prime} k^{\prime}}=a_{k^{\prime}{ }_{1} \cdots k_{m}^{\prime}}^{1 / 2} e_{j^{\prime} k^{\prime}} \zeta^{k^{\prime}}\right)$. Then it is easy to see that

$$
\begin{aligned}
\left\langle g_{j k}, g_{j^{\prime} k^{\prime}}\right\rangle_{D_{P, m}^{\varphi}} & =\int_{D_{P, m}^{\varphi}} g_{j k}(z, \zeta) \overline{g_{j^{\prime} k^{\prime}}(z, \zeta)} d z d \zeta \\
& =\int_{z \in D}\left\langle g_{j k}(z, \cdot), g_{j^{\prime} k^{\prime}}(z, \cdot)\right\rangle_{D_{P}^{m}(\sqrt{\varphi(z)})} d z .
\end{aligned}
$$

Thus $g_{j k}$ and $g_{j^{\prime} k^{\prime}}$ are orthogonal in $L_{a}^{2}\left(D_{P, m}^{\varphi}\right)$ if $k \neq k^{\prime}$. Now assume that $k=k^{\prime}$ and $j \neq j^{\prime}$. Then we have

$$
\begin{aligned}
\left\langle g_{j k}, g_{j^{\prime} k}\right\rangle_{D_{P, m}^{\varphi}} & =\int_{D_{P, m}^{\varphi}} g_{j k}(z, \zeta) \overline{g_{j^{\prime} k}(z, \zeta)} d z d \zeta \\
& =\left\langle e_{j k}, e_{j^{\prime} k}\right\rangle_{L_{a}^{2}\left(D, \varphi^{|\alpha(1+k)|}\right)}=0 .
\end{aligned}
$$

Since the normality is easily verified, we know the set $S$ is an orthonormal set. Assume that $\left\langle f, g_{k j}\right\rangle=0$ for all $j \in J_{k}$ and $k \in \mathbb{N}^{m}$. Then we have

$$
\begin{aligned}
\left\langle f, g_{j k}\right\rangle_{D_{P, m}^{\varphi}} & =\int_{D_{P, m}^{\varphi}} f(z, \zeta) \overline{g_{j k}(z, \zeta)} d z d \zeta \\
& =a_{k_{1} \cdots k_{m}}^{-1 / 2}\left\langle b_{k}(z), e_{j k}\right\rangle_{L_{a}^{2}(D, \varphi|\alpha(1+k)|)}=0,
\end{aligned}
$$

for all $j \in J_{k}$ and $k \in \mathbb{N}^{m}$. Since $b_{k}(z)$ is an element of $L_{a}^{2}\left(D, \varphi^{|\alpha(1+k)|}\right)$ and $\left\{e_{j k}\right\}_{j \in J_{k}}$ is a complete orthonormal basis of $L_{a}^{2}\left(D, \varphi^{|\alpha(1+k)|}\right)$, the condition $\left\langle f, g_{k j}\right\rangle=0$ implies that $b_{k}=0$ for all $k \in \mathbb{N}^{m}$. Therefore the set $S$ is a complete orthonormal basis of $L_{a}^{2}\left(D_{P, m}^{\varphi}\right)$. Hence we finally obtain

$$
\begin{aligned}
K_{D, \varphi|\alpha(1+k)|}\left(z, z^{\prime}\right) & =\sum_{j \in J_{k}} e_{j k}(z) \overline{e_{j k}\left(z^{\prime}\right)}, \\
K_{D_{P, m}^{\varphi}}\left((z, \zeta),\left(z^{\prime}, \zeta^{\prime}\right)\right) & =\sum_{j \in J_{k}, k \in \mathbb{N}^{m}} a_{k_{1} \cdots k_{m}} e_{j k}(z) \overline{e_{j k}\left(z^{\prime}\right)}\left(\overline{\zeta \zeta^{\prime}}\right)^{k} \\
& =\sum_{k \in \mathbb{N}^{m}} a_{k_{1} \cdots k_{m}} K_{D, \varphi}|\alpha(1+k)|
\end{aligned}
$$

This completes the proof of the theorem.

Let us describe how to recover the Forelli-Rudin construction (1.1) of a Hartogs domain. Recall that $D_{P, m}^{\varphi}$ is a Hartogs domain if $P\left(x_{1}, \cdots x_{m}\right)=x_{1}+\cdots+x_{m}$. By 
Example 2.3. we know that $\varphi^{|\alpha(1+k)|}=\varphi^{m+|k|}$. Using Theorem 3.1, we have the following series representation of the Bergman kernel $K$ of the Hartogs domain:

$$
\begin{aligned}
K\left((z, \zeta),\left(z^{\prime}, \zeta^{\prime}\right)\right) & =\sum_{k_{1}, \cdots, k_{m} \geq 0} \frac{(|k|+m) !}{\pi^{m} k_{1} ! \cdots k_{m} !} K_{D, \varphi^{|k|+m}}\left(z, z^{\prime}\right)\left(\overline{\zeta_{1} \bar{\zeta}_{1}}\right)^{k_{1}} \cdots\left(\overline{\zeta_{m}} \overline{\zeta_{m}^{\prime} m}\right)^{k_{m}} \\
& =\frac{1}{\pi^{m}} \sum_{k \geq 0}(k+1)_{m} K_{D, \varphi^{k+m}}\left(z, z^{\prime}\right)\left\langle\zeta, \zeta^{\prime}\right\rangle^{k} .
\end{aligned}
$$

Here the second equality follows from the following simple identity:

$$
\frac{\left(x_{1}+\cdots+x_{m}\right)^{k}}{k !}=\sum_{|\alpha|=k} \frac{x^{\alpha}}{\alpha !} .
$$

Thus the Forelli-Rudin construction (1.1) for the Hartogs domain is recovered.

We next consider the following case:

$$
D_{q, m}^{\varphi}=\left\{(z, \zeta) \in D \times \mathbb{C}^{m} ;\left|\zeta_{1}\right|^{2 q_{1}}+\cdots+\left|\zeta_{m}\right|^{2 q_{m}}<\varphi(z)\right\} .
$$

By Theorem 3.1 and Example 2.4 we have the following corollary:

Corollary 3.2. The Bergman kernel of $D_{q, m}^{\varphi}$ has the following series representation:

$$
K_{D_{q, m}^{\varphi}}\left((z, \zeta),\left(z^{\prime}, \zeta^{\prime}\right)\right)=\sum_{k \in \mathbb{N}^{m}}^{\infty} a_{k_{1} \cdots k_{m}} K_{D, \varphi^{\left|q^{-1}(1+k)\right|}}\left(z, z^{\prime}\right)\left(\zeta \bar{\zeta}^{\prime}\right)^{k},
$$

where $a_{k_{1} \cdots k_{m}}$ is given by (2.7).

3.2. Roos' problem. Here we discuss further generalizations of Corollary 3.2 and related results. Let $q_{i}$ be a positive integer and $\varphi_{i}$ a positive continuous function on $D$ for $1 \leq i \leq m$. Consider the following domain:

$$
\widetilde{D}_{q, m}^{\varphi}:=\left\{(z, \zeta) \in D \times \mathbb{C}^{m} ; \sum_{i=1}^{m} \frac{\left|\zeta_{i}\right|^{2 q_{i}}}{\varphi_{i}(z)}<1\right\} .
$$

The domain $\widetilde{D}_{q, m}^{\varphi}$ includes as a special case the domain $D_{q, m}^{\varphi}$. In a completely analogous way to the proof of Theorem [3.1, we can prove a Forelli-Rudin type formula for $\widetilde{D}_{q, m}^{\varphi}$. To avoid the repetition, we only formulate the theorem omitting its proof.

Theorem 3.3. The Bergman kernel $K_{\widetilde{D}_{q, m}^{\varphi}}$ of $\widetilde{D}_{q, m}^{\varphi}$ has the following series representation:

$$
K_{\widetilde{D}_{q, m}^{\varphi}}\left((z, \zeta),\left(z^{\prime}, \zeta^{\prime}\right)\right)=\sum_{k \in \mathbb{N}^{m}} a_{k_{1} \cdots k_{m}} K_{m, q, k}\left(z, z^{\prime}\right)\left(\zeta \overline{\zeta \zeta^{\prime}}\right)^{k},
$$

where $a_{k_{1} \cdots k_{m}}$ is given by (2.7) and the kernel function $K_{m, q, k}$ is the weighted Bergman kernel of $D$ with respect to a weight $\prod_{i=1}^{m} \varphi_{i}(z)^{\frac{1+k_{i}}{q_{i}}}$.

Recall that the Hartogs domain can be rewritten as

$$
D_{m, \varphi}=\left\{(z, \zeta) \in D \times \mathbb{C}^{m} ; \varphi(z)^{-1 / 2} \zeta \in \mathbb{B}^{m}\right\} .
$$

Moreover, the Forelli-Rudin construction (1.1) can be rewritten as

$$
K_{D_{m, \varphi}}\left((z, \zeta),\left(z^{\prime}, \zeta^{\prime}\right)\right)=\left.\frac{1}{\pi^{m}} \frac{\partial^{m}}{\partial r^{m}} L_{D, \varphi}\left(z, z^{\prime} ; r\right)\right|_{r=\left\langle\zeta, \zeta^{\prime}\right\rangle} .
$$


Here the function $L_{D, \varphi}\left(z, z^{\prime} ; r\right)$ is given by

$$
L_{D, \varphi}\left(z, z^{\prime} ; r\right)=\sum_{k=0}^{\infty} K_{D, \varphi^{k}}\left(z, z^{\prime}\right) r^{k},
$$

and it is called the virtual Bergman kernel of $(D, \varphi)$. In [18, G. Roos introduced the following domain $D_{\varphi}^{\Omega}$, which is a generalization of the right hand side of the above expression (3.2):

$$
D_{\varphi}^{\Omega}:=\left\{(z, \zeta) \in D \times \mathbb{C}^{m} ; \varphi(z)^{-1 / 2} \zeta \in \Omega\right\},
$$

where $\Omega \subset \mathbb{C}^{m}$ is a domain. Then he put forth the following problem [18, Problem 4.2]:

Problem 3.4. (1) If $\Omega$ is any circled domain in $\mathbb{C}^{m}$, what can be said about the Bergman kernel of $D_{\varphi}^{\Omega}$ ? (2) For some families $\{F\}$ other than the family $\left\{\mathbb{B}^{m}\right\}$ of Hermitian balls, is it possible to define an analogue of the virtual Bergman kernel $L_{\Omega, \varphi}$ and obtain an analogue of (3.3)?

M. Engliš and G. Zhang [5] answered this problem when $F$ is a bounded symmetric domain. Namely, they obtained a Forelli-Rudin type formula of $D_{\varphi}^{\Omega}$ when $\Omega$ is an irreducible bounded symmetric domain and also obtained an analogue of (3.3) when $\Omega$ is an irreducible bounded symmetric domain of Type I or Type IV. Recently, the domain $D_{\varphi}^{\Omega}$ was also investigated by Z. Feng [6] in a different context. Feng introduced invariant Hilbert spaces of holomorphic functions on $D_{\varphi}^{\Omega}$ and computed their reproducing kernels explicitly.

As an application of Theorem 3.3, we derive a Forelli-Rudin type formula for the domain $D_{\varphi}^{\Omega}$ when $\Omega$ is the complex ellipsoid $D_{q}$ of radius 1 , which is not bounded symmetric in general:

Theorem 3.5. The Bergman kernel $K$ of $D_{\varphi}^{\Omega}$ has the following series representation when $\Omega$ is the complex ellipsoid $D_{q}$ of radius 1 :

$$
K\left((z, \zeta),\left(z^{\prime}, \zeta^{\prime}\right)\right)=\sum_{k \in \mathbb{N}^{m}} a_{k_{1} \cdots k_{m}} K_{D, \varphi^{m+|k|}}\left(z, z^{\prime}\right)\left(\zeta \overline{\zeta^{\prime}}\right)^{k},
$$

where $a_{k_{1} \cdots k_{m}}$ is given by (2.7).

Proof. Put $\varphi_{i}=\varphi^{q_{i}}$ for any $1 \leq i \leq m$. Then we know that

$$
\begin{aligned}
\widetilde{D}_{q, m}^{\varphi} & =\left\{(z, \zeta) \in D \times \mathbb{C}^{m} ; \sum_{i=1}^{m} \frac{\left|\zeta_{i}\right|^{2 q_{i}}}{\varphi(z)^{q_{i}}}<1\right\} \\
& =\left\{(z, \zeta) \in D \times \mathbb{C}^{m} ; \varphi(z)^{-\frac{1}{2}} \zeta \in D_{q}\right\} .
\end{aligned}
$$

Thus the domain $\widetilde{D}_{q, m}^{\varphi}$ coincides with $D_{\varphi}^{\Omega}$. In this case, the weight function $\prod_{i=1}^{m} \varphi_{i}(z)^{\frac{k_{i}+1}{q_{i}}}$ is given by

$$
\prod_{i=1}^{m} \varphi_{i}(z)^{\frac{k_{i}+1}{q_{i}}}=\varphi^{\sum_{i=1}^{m} q_{i}\left(\frac{k_{i}+1}{q_{i}}\right)}=\varphi(z)^{m+|k|} .
$$

Thus the proof is complete.

This answers Problem 3.4(1) when $\Omega$ is the complex ellipsoid $D_{q}$. However we do not succeed in obtaining an analogue of (3.3). 


\section{Deflation iDENTITY}

Let $\Omega_{1}, \Omega_{2}$ be domains in $\mathbb{C}^{m_{1}}$ and in $\mathbb{C}^{m_{2}}$ respectively. In general, there is no relation between the Bergman kernels $K_{\Omega_{1}}$ and $K_{\Omega_{2}}$. However, as explained in Section 1, if we consider two domain $\Omega_{1}$ and $\Omega_{2}$ as in Section 1.2 then the restrictions of the Bergman kernels of $\Omega_{1}$ and $\Omega_{2}$ to the subspace $\left\{\zeta, \zeta^{\prime}=0\right\}$ coincide with each other (up to a constant multiple).

In this section, as an application of our results, we study deflation identities for $D_{P, m}^{\varphi}$. We start our study with the observation that the restriction of the Bergman kernel of $D_{P, m}^{\varphi}$ to the subspace $\left\{\zeta, \zeta^{\prime}=0\right\}$ represents the weighted Bergman kernel $K_{D, \varphi|\alpha|}$. Indeed, as a consequence of Theorem 3.1, we have the following lemma.

Lemma 4.1. The restriction of the Bergman kernel of $D_{P, m}^{\varphi}$ to the subspace $\left\{\zeta, \zeta^{\prime}=0\right\}$ is given by

$$
K_{D_{P, m}^{\varphi}}\left((z, 0),\left(z^{\prime}, 0\right)\right)=a_{0} K_{D, \varphi|\alpha|}\left(z, z^{\prime}\right) .
$$

Before stating our result, it is convenient to introduce the following equivalence relation:

Definition 4.2. Let $r_{i}$ be a positive real number for $i=1,2$. We say that the domains $D_{P, m}^{\varphi^{r_{1}}}$ and $D_{P^{\prime}, m^{\prime}}^{\varphi^{r_{2}}}$ are equivalent if $r_{1}|\alpha|=r_{2}\left|\alpha^{\prime}\right|$.

Let $K_{1}$ (resp. $\left.K_{2}\right)$ be the Bergman kernel of $D_{P, m_{1}}^{\varphi^{r_{1}}}\left(\operatorname{resp} . D_{P^{\prime}, m_{2}}^{\varphi^{r_{2}}}\right)$. We can now prove the following deflation identity:

Theorem 4.3. Assume that the domains $D_{P, m_{1}}^{\varphi^{r_{1}}}$ and $D_{P^{\prime}, m_{2}}^{\varphi^{r_{2}}}$ are equivalent. Then the restrictions of the Bergman kernels $K_{1}, K_{2}$ to the subspace $\left\{\zeta, \zeta^{\prime}=0\right\}$ coincide with each other (up to a constant multiple):

$$
c_{1} K_{1}\left((z, 0),\left(z^{\prime}, 0\right)\right)=c_{2} K_{2}\left((z, 0),\left(z^{\prime}, 0\right)\right),
$$

where $c_{1}$ and $c_{2}$ are constants.

Proof. By Lemma 4.1, we have

$$
K_{i}\left((z, 0),\left(z^{\prime}, 0\right)\right)=a_{0}^{(i)} K_{D, \varphi^{r_{i}|\alpha|}}\left(z, z^{\prime}\right) .
$$

Since $D_{P, m_{1}}^{\varphi^{r_{1}}}$ and $D_{P^{\prime}, m_{2}}^{\varphi^{r_{2}}}$ are equivalent, we conclude that

$$
\begin{aligned}
K_{1}\left((z, 0),\left(z^{\prime}, 0\right)\right) & =a_{0}^{(1)} K_{D, \varphi^{r_{1}|\alpha|}}\left(z, z^{\prime}\right) \\
& =a_{0}^{(1)} K_{D, \varphi^{r_{2}\left|\alpha^{\prime}\right|}}\left(z, z^{\prime}\right)=\frac{a_{0}^{(1)}}{a_{0}^{(2)}} K_{2}\left((z, 0),\left(z^{\prime}, 0\right)\right) .
\end{aligned}
$$

Thus the proof is complete.

Now let us turn back to the original deflation identity (1.2) of the following domains $\Omega_{1}, \Omega_{2}$ :

$$
\begin{aligned}
& \Omega_{1}=\left\{(z, \zeta) \in \Omega \times \mathbb{C} ;|\zeta|^{2}<(1-\psi(z))^{q_{1}+q_{2}}\right\}, \\
& \Omega_{2}=\left\{(z, \zeta) \in \Omega \times \mathbb{C}^{2} ;\left|\zeta_{1}\right|^{2 / q_{1}}+\left|\zeta_{2}\right|^{2 / q_{2}}<1-\psi(z)\right\} .
\end{aligned}
$$

To recover the deflation identity (1.2) from Theorem 4.3, it is enough to check that $\Omega_{1}$ and $\Omega_{2}$ are equivalent. Actually the equivalence of $\Omega_{1}$ and $\Omega_{2}$ is obvious from 
the definitions. Moreover, the following domains are also equivalent to $\Omega_{1}$ and $\Omega_{2}$ :

$$
\begin{aligned}
& \Omega_{3}=\left\{(z, \zeta) \in \Omega \times \mathbb{C}^{2} ;\left|\zeta_{1}\right|^{2}+\left|\zeta_{2}\right|^{2 / q_{2}}<(1-\psi(z))^{\frac{q_{1}+q_{2}}{1+q_{2}}}\right\}, \\
& \Omega_{4}=\left\{(z, \zeta) \in \Omega \times \mathbb{C}^{2} ;\left|\zeta_{1}\right|^{2 / q_{1}}+\left|\zeta_{2}\right|^{2 / q_{1}}<(1-\psi(z))^{\frac{q_{1}+q_{2}}{2 q_{1}}}\right\} .
\end{aligned}
$$

In summary, we have

Corollary 4.4. The restrictions of the Bergman kernels $K_{\Omega_{i}}, K_{\Omega_{j}}$ to the subspace $\left\{\zeta, \zeta^{\prime}=0\right\}$ coincide with each other (up to a constant multiple) for any $1 \leq i, j \leq 4$.

Thus we have recovered the deflation identity (1.2). Next, we turn to studying deflation identities in a more general setting. Consider the following domains:

$$
\begin{aligned}
& D_{p, m_{1}}^{\varphi^{r_{1}}}:=\left\{(z, \zeta) \in D \times \mathbb{C}^{m_{1}} ;\left|\zeta_{1}\right|^{2 / p_{1}}+\cdots+\left|\zeta_{m_{1}}\right|^{2 / p_{m_{1}}}<\varphi(z)^{r_{1}}\right\}, \\
& D_{q, m_{2}}^{\varphi^{r_{2}}}:=\left\{(z, \zeta) \in D \times \mathbb{C}^{m_{2}} ;\left|\zeta_{1}\right|^{2 / q_{1}}+\cdots+\left|\zeta_{m_{2}}\right|^{2 / q_{m_{2}}}<\varphi(z)^{r_{2}}\right\} .
\end{aligned}
$$

Then these domains $D_{p, m_{1}}^{\varphi^{r_{1}}}, D_{q, m_{1}}^{\varphi^{r_{2}}}$ are equivalent if and only if $r_{1}|p|=r_{2}|q|$. Let $K_{1}$ (resp. $K_{2}$ ) denote the Bergman kernel of $D_{p, m_{1}}^{\varphi^{r_{1}}}$ (resp. $D_{q, m_{1}}^{\varphi^{r_{2}}}$ ). Consequently, we obtain the following identity between $K_{1}$ and $K_{2}$.

Corollary 4.5. Assume that $r_{1}|p|=r_{2}|q|$. Then the restrictions of the Bergman kernels $K_{1}, K_{2}$ to the subspace $\left\{\zeta, \zeta^{\prime}=0\right\}$ coincide with each other (up to a constant multiple).

Remark 4.6. We remark that in the case of the Hartogs domain, the condition $r_{1}|p|=r_{2}|q|$ is more restrictive. Consider two Hartogs domains:

$$
D_{m_{i}, \varphi^{r_{i}}}=\left\{(z, \zeta) \in D \times \mathbb{C}^{m_{i}} ;\|\zeta\|^{2}<\varphi(z)^{r_{i}}\right\}, \quad i=1,2 .
$$

It is easily verified that $D_{m_{1}, \varphi^{r_{1}}}$ and $D_{m_{2}, \varphi^{r_{2}}}$ are equivalent if and only if $r_{1} m_{1}=$ $r_{2} m_{2}$. Therefore if $r_{1}=r_{2}$ and $m_{1} \neq m_{2}$, then $D_{m_{1}, \varphi^{r_{1}}}$ and $D_{m_{2}, \varphi^{r_{2}}}$ are not equivalent. On the contrary, in the case of $D_{p, m_{1}}^{\varphi^{r_{1}}}$ and $D_{q, m_{2}}^{\varphi^{r_{2}}}$, there is the possibility $D_{p, m_{1}}^{\varphi^{r_{1}}} \sim D_{q, m_{2}}^{\varphi^{r_{2}}}$ even if we assume that $r_{1}=r_{2}$ and $m_{1} \neq m_{2}$.

Using Theorem 3.1 and Theorem 3.5, we also obtain deflation identities between $D_{P, m}^{\varphi}$ and $D_{\varphi}^{\Omega}$ in an analogous way. For instance, we have a deflation identity between the Hartogs domain $D_{m, \varphi}$ and $D_{\varphi}^{\Omega}$.

Theorem 4.7. Let $\Omega$ be a complex ellipsoid of radius 1 . Then the restrictions of the Bergman kernels $K_{D_{m, \varphi}}, K_{D_{\varphi}^{\Omega}}$ to the subspace $\left\{\zeta, \zeta^{\prime}=0\right\}$ coincides with each other (up to a constant multiple).

Since the proof proceeds along the same lines as that of Theorem 4.3, we omit it.

\section{ACKNOWLEDGEMENTS}

The author is grateful to Professor Kang-Tae Kim for stimulating discussions and valuable comments on this study. We also thank the referee for a careful reading of our manuscript and for giving useful comments. 


\section{REFERENCES}

[1] Harold P. Boas, Siqi Fu, and Emil J. Straube, The Bergman kernel function: explicit formulas and zeroes, Proc. Amer. Math. Soc. 127 (1999), no. 3, 805-811, DOI 10.1090/S0002-993999-04570-0. MR1469401 (99f:32037)

[2] John P. D'Angelo, A note on the Bergman kernel, Duke Math. J. 45 (1978), no. 2, 259-265. MR0473231 (57 \#12906)

[3] Miroslav Engliš, Zeroes of the Bergman kernel of Hartogs domains, Comment. Math. Univ. Carolin. 41 (2000), no. 1, 199-202. MR.1756941 (2001f:32002)

[4] Miroslav Engliš, A Forelli-Rudin construction and asymptotics of weighted Bergman kernels, J. Funct. Anal. 177 (2000), no. 2, 257-281, DOI 10.1006/jfan.2000.3642. MR 1795632 (2001k:32004)

[5] Miroslav Engliš and Genkai Zhang, On a generalized Forelli-Rudin construction, Complex Var. Elliptic Equ. 51 (2006), no. 3, 277-294, DOI 10.1080/17476930500515017. MR2200983 (2007a:32001)

[6] Feng ZhiMing, Hilbert spaces of holomorphic functions on generalized CartanHartogs domains, Complex Var. Elliptic Equ. 58 (2013), no. 3, 431-450, DOI 10.1080/17476933.2011.598927. MR3011934

[7] Frank Forelli and Walter Rudin, Projections on spaces of holomorphic functions in balls, Indiana Univ. Math. J. 24 (1974/75), 593-602. MR0357866 (50 \#10332)

[8] Gábor Francsics and Nicholas Hanges, The Bergman kernel of complex ovals and multivariable hypergeometric functions, J. Funct. Anal. 142 (1996), no. 2, 494-510, DOI 10.1006/jfan.1996.0157. MR.1423042 (97m:32039)

[9] Siqi Fu, A. V. Isaev, and Steven G. Krantz, Reinhardt domains with non-compact automorphism groups, Math. Res. Lett. 3 (1996), no. 1, 109-122, DOI 10.4310/MRL.1996.v3.n1.a11. MR.1393388 (97c:32043)

[10] Robert E. Greene, Kang-Tae Kim, and Steven G. Krantz, The geometry of complex domains, Progress in Mathematics, vol. 291, Birkhäuser Boston Inc., Boston, MA, 2011. MR2799296 (2012c:32001)

[11] A. V. Isaev and S. G. Krantz, Finitely smooth Reinhardt domains with non-compact automorphism group, Illinois J. Math. 41 (1997), no. 3, 412-420. MR1458181 (98i:32054)

[12] A. V. Isaev and S. G. Krantz, Domains with non-compact automorphism group: a survey, Adv. Math. 146 (1999), no. 1, 1-38, DOI 10.1006/aima.1998.1821. MR1706680(2000i:32053)

[13] Steven G. Krantz, Function theory of several complex variables, 2nd ed., The Wadsworth \& Brooks/Cole Mathematics Series, Wadsworth \& Brooks/Cole Advanced Books \& Software, Pacific Grove, CA, 1992. MR:1162310 (93c:32001)

[14] Ewa Ligocka, On the Forelli-Rudin construction and weighted Bergman projections, Studia Math. 94 (1989), no. 3, 257-272. MR.1019793 (90i:32034)

[15] Jong-Do Park, New formulas of the Bergman kernels for complex ellipsoids in $\mathbb{C}^{2}$, Proc. Amer. Math. Soc. 136 (2008), no. 12, 4211-4221, DOI 10.1090/S0002-9939-08-09576-2. MR2431034(2009d:32001)

[16] Jong-Do Park, Explicit formulas of the Bergman kernel for 3-dimensional complex ellipsoids, J. Math. Anal. Appl. 400 (2013), no. 2, 664-674, DOI 10.1016/j.jmaa.2012.11.017. MR.3004995

[17] R. Michael Range, Holomorphic functions and integral representations in several complex variables, Graduate Texts in Mathematics, vol. 108, Springer-Verlag, New York, 1986. MR.847923(87i:32001)

[18] Guy Roos, Weighted Bergman kernels and virtual Bergman kernels, Sci. China Ser. A 48 (2005), no. suppl., 225-237, DOI 10.1007/BF02884708. MR2156503(2006e:32007)

[19] Satoru Shimizu, Automorphisms of bounded Reinhardt domains, Japan. J. Math. (N.S.) 15 (1989), no. 2, 385-414. MR 1039248 (91j:32001)

[20] Toshikazu Sunada, Holomorphic equivalence problem for bounded Reinhardt domains, Math. Ann. 235 (1978), no. 2, 111-128. MR0481064 (58 \#1211)

[21] A. Yamamori, The Bergman kernel of the Fock-Bargmann-Hartogs domain and the polylogarithm function, Complex Var. Elliptic Eqs., 58 (2013), no. 6, 783-793. MR3170660

[22] Atsushi Yamamori, A remark on the Bergman kernels of the Cartan-Hartogs domains (English, with English and French summaries), C. R. Math. Acad. Sci. Paris 350 (2012), no. 3-4, 157-160, DOI 10.1016/j.crma.2012.01.005. MR2891103 
[23] Atsushi Yamamori, A note on the Bergman kernel of a certain Hartogs domain (English, with English and French summaries), C. R. Math. Acad. Sci. Paris 350 (2012), no. 17-18, 827-829, DOI 10.1016/j.crma.2012.10.009. MR.2989385

[24] Yunus E. Zeytuncu, Weighted Bergman projections and kernels: $L^{p}$ regularity and zeros, Proc. Amer. Math. Soc. 139 (2011), no. 6, 2105-2112, DOI 10.1090/S0002-9939-2010-107955. MR2775388 (2012d:30135)

Center for Geometry and its Applichtions and Department of Mathematics, POSTECH, Pohang City 790-784, The Republic of Korea

E-mail address: yamamori@postech.ac.kr, ats.yamamori@gmail.com 